\title{
Novel, statistical approach for structure determination of modulated pathogenesis-related protein (Hyp-1) complex with ANS
}

\author{
J.Smietanska ${ }^{1}$, J. Sliwiak ${ }^{2}$, M. Jaskolski ${ }^{2}$, M. Gilski ${ }^{2}$, Z. Dauter ${ }^{3}$, R. Strzalka ${ }^{1}$, J.Wolny ${ }^{1}$ \\ ${ }^{1}$ AGH University of Science and Technology, al. Mickiewicza 30,, 30-059 Krakow, Poland, \\ ${ }^{2}$ Center for Biocrystallographic Research, Institute of Bioorganic Chemistry, Polish Academy of Sciences, Zygmunta Noskowskiego \\ 12/14, 61-704 Poznan, Poland, \\ ${ }^{3}$ Synchrotron Radiation Research Section, MCL, National Cancer Institute, Argonne National Laboratory, Argonne IL 60439, USA
}

joanna.smietanska@fis.agh.edu.pl

Newly discovered, and still uncommon, modulated crystal structure in organic systems require a deeper investigation. No exact and detailed solution of such systems has not been done up-to-date. One possibility is to use an approximation of commensurate modulation which enables constructing a supercell, extending to the case, where translational symmetry (periodicity) is recovered, and simplify the analysis [1]. An assumption of commensurateness of the modulation is, however, questionable and rather unverifiable.

The goal of our studies was to use a novel, original statistical method of structural modeling which enables a refinement based on the average unit cell with (commensurate or incommensurate) modulation without unclear assumption of commensurateness and supercell approach. The main concept of the statistical method is to express structure in terms of the statistical distribution of atomic positions concerning the periodic reference lattice with lattice constant related to characteristic length-scale present in the structure. The average unit cell, defined as a probability distribution, constructed for periodic crystal is the same as the unit cell. The statistical approach was successfully used for the description of not only periodic crystals or quasicrystals, as well as it can be expanded on modulated structures as well as aperiodic structures with singular continuous components in the diffraction pattern [2].

Our model system is a pathogenesis-related protein (Hyp-1) complex with fluorescent probe 8-anilino-1-naphthalene sulfonate (ANS), which is a unique example of a macromolecular system with the modulated crystal structure. Previous studies have shown that Hyp1/ANS complexes are tetartohedral twinned and crystallized in an asymmetric unit cell containing a repetitive motif of four protein molecules arranged with 7-fold noncrystallographic repetition along the $c$ axis of the $\mathrm{C} 2$ space group. Assumption of commensurate structure modulation demanded description of structure in the highly expanded unit cell with 28 unique protein molecules inside [3]. The Hyp-1/ANS structure was solved by molecular replacement and refined using maximum-likelihood targets with reliability factors $\mathrm{R}_{\text {work }} / \mathrm{R}_{\text {free }}$ of $22.3 / 27.8 \%$, respectively.

Our approach involved re-integration of raw data, development of the original software in Matlab environment and multidimensional analysis used to build the structure model and perform the refinement for significant improvement of results. The problem of incorporating disorder in the form of phonons into structural analysis was also carried out traditionally by Debye-Waller factor.

[1] Sliwiak, J., Dauter, Z., McCoy, A., Jaskolski, M. \& Read, R.J. (2014). Acta Cryst. D70, 471-480.

[2] Wolny, J., Buganski, I., Kuczera, P. \& Strzalka, R. (2016). J. Appl. Crystallogr. 49, 2106-2115.

[3] Sliwiak, J., Dauter, Z., Kowiel, M., McCoy, A., Read, R.J \& Jaskolski, M. (2015). Acta Cryst. D71, 829-843.

Keywords: protein; modulated structure; aperiodic

Joanna Smietanska has been partly supported by the EU Project POWR.03.02.00-00-I004/16. 